Article

\title{
Universally Balanced Combinatorial Optimization Games
}

\section{Gabrielle Demange ${ }^{1}$ and Xiaotie Deng ${ }^{2, \star}$}

${ }^{1}$ Paris School of Economics, 48 bd Jourdan, 75014 Paris, France; E-Mail: demange@pse.ens.fr

2 Department of Computer Science, City University of Hong Kong, Tat Chee Avenue, Kowloon, Hong Kong

^ Author to whom correspondence should be addressed; E-Mail: deng@cs.cityu.edu.hk.

Received: 27 May 2010; in revised form: 18 August 2010 / Accepted: 23 August 2010 /

Published: 13 September 2010

\begin{abstract}
This article surveys studies on universally balanced properties of cooperative games defined in a succinct form. In particular, we focus on combinatorial optimization games in which the values to coalitions are defined through linear optimization programs, possibly combinatorial, that is subject to integer constraints. In economic settings, the integer requirement reflects some forms of indivisibility. We are interested in the classes of games that guarantee a non-empty core no matter what are the admissible values assigned to the parameters defining these programs. We call such classes universally balanced. We present characterization and complexity results on the universally balancedness property for some classes of interesting combinatorial optimization games. In particular, we focus on the algorithmic properties for identifying universally balancedness for the games under discussion.
\end{abstract}

Keywords: combinatorial cooperative games; balanced; blocking; core; integrality

\section{Introduction}

The universally balanced concept and its properties has been studied, under various names and in different settings (Kaneko and Wooders [1], Faigle and Kern [2], Boros, Gurvich, and Vasin [3] among others). This article proposes a unified framework to present those types of results. The main idea underlying universal balancedness is to identify structural properties that guarantee stability in a cooperative environment. That is, we are interested in structures under which organizations or coalitions 
can find a stable arrangement no matter what specific numerical values are assumed by the parameters defining the game.

Of particular interest is the concept of the core, which describes all distributions of the collective gain among the players such that no subset of players would be able to reach, on their own, a higher value than the current sum allocated to them. The study could also be conducted on other solution concepts in cooperative games. Our choice reflects personal familiarity with the concept, and also the fact that the core has the main characteristics that any possibly rational concept should possess.

We conduct our analysis in cooperative games for which a succinct representation is possible. This is a very useful consideration in terms of the computational complexity. The computational issues are becoming an indispensable component in game theory for practical implementation. Take the core as an example. For $n$ players, the game is defined by assigning a value to each subset of the $n$ players (coalition). Therefore, in principle, one needs to handle $2^{n}$ coalitions, which is an exponential size in the number of players. Such a high level of complexity may be unnecessary, and calls for a succinct description of a game, as suggested by Megiddo [4]. To be specific, we consider games for which the values to coalitions are defined as the values of linear optimization problems, possibly combinatorial, that is subject to integer constraints. Many important applications are modeled that way. Two prominent examples are the production game introduced by Owen [5], and the assignment game introduced by Shapley and Shubik [6], which is combinatorial.

Universally balancedness bears on games that are described by (possibly multi-dimensional) parameters, the values of some resources or prices for instance. A class of games is universally balanced with respect to some parameter if the game has a non-empty core whatever values this parameter can assume. The class of assignment games, as an example, is universally balanced with respect to the weight on the edges of the defining graph. We are interested in characterization results for such games, as well as computational complexities identifying them.

We organize the article as follows. Section 2 presents some examples, the model, the concept of universally balanced, and a short discussion about complexity issues in cooperative games. Section 3 presents some general results about universally balanced classes and the impact of the integer constraint on the cores of a game. Section 4 provides a characterization and computational complexity results for the universally balanced property in a special class of combinatorial optimization games, called simple. We illustrate the results using a simple restricted version of the problems on graphs. Section 5 is a preliminary analysis of the universally balanced property with respect to the feasibility constraints in production games. Section 6 concludes.

\section{Model and Definitions}

A (transferable utility) cooperative game, $\operatorname{Game}(\nu, N)$, is commonly defined by its players set, $N$, and the characteristic function $\nu$ that assigns a value $\nu(S)$ to every coalition (subset of $N$ ); hence $\nu$ : $2^{N} \rightarrow R$. The game is said to be super-additive if the sum of the value of two disjoint coalitions is not larger than the value of their union, that is, if it satisfies the property : $\nu(T)+\nu(S) \leq \nu(S \cup T)$ for $S$ and $T \subseteq N$ disjoint. In what follows we consider super-additive games. ${ }^{1}$

\footnotetext{
${ }^{1}$ In the absence of interactions between disjoint coalitions, super-additivity is not a restriction. If $\nu$ is not super-additive, the value of a coalition can be redefined as the maximum of the value of its partitions, as in a partitioning game defined below.
} 
The outcome of a cooperative game is an allocation described by a vector $z \in R^{N}$ that specifies the payoff $z_{i}$ to each player $i$. To be feasible, it must satisfy $\sum_{i \in N} z_{i} \leq \nu(N)$. An imputation is an allocation that satisfies individual rationality: $\forall i, z_{i} \geq \nu(\{i\})$ and efficiency: $\sum_{i \in N} z_{i}=\nu(N)$. Individual rationality and efficiency are two minimal requirements. The core is the set of imputations that satisfy further conditions, called the no-blocking conditions or the sub-group rationality conditions. A coalition $S$ blocks $z$ if $z(S)<\nu(S)$ where $z(S)=\sum_{i \in S} z_{i}$ denotes the sum of the payoffs to the members of $S$. The rationale is that the players in $S$ can be made all strictly better off than in $z$ by making an arrangement among themselves and dividing appropriately the value $\nu(S)$. The core is thus defined by

$$
\operatorname{core}(\nu, N)=\left\{z \in R^{N}, z(N)=\nu(N), z(S) \geq \nu(S) \forall S \subseteq N\right\}
$$

Similarly cost sharing games are defined by assigning a cost to each coalition: $\sigma: 2^{N} \rightarrow R$. For example, the cost $c(S)$ is interpreted as the cost for $S$ of providing some services to its members. The previous definition are easily adapted as follows by viewing a cost as the opposite of a value. A vector $z \in R^{N}$ is in the core, $\operatorname{core}(\sigma, N)$, if and only if it satisfies efficiency, $z(N)=\sigma(N)$ and the no-blocking conditions: $z(S) \leq \sigma(S)$ for all $S \subseteq N$.

Let us say a few words about the computational aspects for this problem. A computational problem is defined by its "inputs" and the solution to be computed. The input for a cooperative game includes the set $N$ and the function $\nu$. As $\nu$ is defined on $2^{N}$, it requires in general $2^{|N|}$ values, one for each subset $S \subseteq N$. The input size will already be an exponential function in the number of players. Under such circumstance, any solution concept (unless the very trivial ones) would require time exponential in the number of players. This is true for the core. To evaluate whether an allocation satisfies the sub-group rationality constraints of the core requires an exponential number of linear inequalities. In many practical settings however, such as in the models we are going to introduce, such a high number is not necessary (at least for the input).

We introduce three classes of games, respectively called production, cost sharing and partitioning games. These games differ by the type of constraints that define the values to coalitions. Roughly speaking, a production game deals with resource constraints, a cost sharing game with demand constraints, and a partitioning game with exact utilization constraints. For each game, we consider a version with "indivisibilities" in which the variables that can be chosen by a coalition are restricted to be integer valued.

For such models, it is convenient to use linear programming formulation to define the values to subsets of players in the game, where the objective function is a linear function of the activity variables, and the constraints are productivity conditions (or consumption conditions) on the variables. The indivisibility of goods are, as pointed above, enforced by the integrality conditions on variables.

The new game-called the super-additive cover-is super-additive and represents the same possibilities of cooperation as the initial game. For a discussion on cooperative games see Demange and Wooders [7], part 2 for instance. 


\subsection{Examples}

We first introduce some examples that motivated our study.

\subsubsection{Owen's linear production model}

Owen [5] considers a situation in which each of the $n$ players is endowed with some resources that are used as factors of production. Linear production technologies transform these resources into final goods that can be sold at a given market price. All coalitions have access to these technologies.

There are $m$ resources and $p$ final goods. A $m \times p$ matrix $A$ describes the technologies. Specifically, a unit of the final good $j, j=1, \cdots, p$, requires $A_{k, j}$ units of resource $k, k=1, \cdots, m$. The price of good $j$ is $v_{j}$. The endowments of the $n$ players are described by the units $b_{k}^{i}$ in resource $k, k=1, \cdots, m$, for each player $i, i=1, \cdots, n$. To sum up, the data are described by $(A, v, b)$. The game is built on linear optimization problems based on these data.

The members of a coalition $S$ can put their resources in common. The value of coalition $S$ is defined as the maximum market value of the outputs the members of $S$ can achieve when they put their resources in common. Letting $x \in R^{p}$ be the outputs produced by a coalition, the value to $S \subset N$ is defined as the solution to the following linear program:

$$
\begin{aligned}
\nu_{L}(S)=\max & v^{t} x \\
\text { s.t. } & A x \leq b(S), x \geq 0
\end{aligned}
$$

where $b(S)=\left(b_{1}(S), b_{2}(S), \cdots, b_{m}(S)\right)^{t}$ and $b_{k}(S)=\sum_{i \in S} b_{k}^{i}$ and we use the notation $v^{t} x=\sum_{j} v_{j} x_{j}$.

The characteristic function $\nu_{L}$ is super-additive. The core problem is to allocate the revenues $\nu_{L}(N)$ in such a way that no coalition could achieve higher revenue to each of its members. The core is non empty, as shown by Owen [5] (see also Theorem 1).

\subsubsection{Linear cost sharing game}

Similarly to the production model, we define a linear cost sharing game in which individuals have some demands or minimal needs to be satisfied. Let $d_{k}^{i}$ be $i$ 's demand of $k$. The unit cost of each $j$ is $c_{j}$ and the use of $x=\left(x_{j}\right) \in R^{p}$ yields the output vector $A x$. An interpretation is that $j$ represents a technology which yields a joint production described by the column $j$ of matrix $A$.

The cost to $S \forall S \subseteq N, \sigma_{L}(S)$ is defined by :

$$
\begin{aligned}
& \sigma_{L}(S)=\min \quad c^{t} x \\
& \text { s.t. } \quad A x \geq d(S) \quad, x \geq 0
\end{aligned}
$$

where $d(S)=\left(d_{1}(S), d_{2}(S), \cdots, d_{m}(S)\right)^{t}$ and $d_{k}(S)=\sum_{i \in S} d_{k}^{i}$.

Function $\sigma_{L}$ is sub-additive, that is, the sum of the cost for two disjoint coalitions is not smaller than the cost for their union. The core problem is to allocate the costs $\sigma_{L}(N)$ in such a way that no coalition could achieve the demands of its members at a lower cost. The core is non-empty (see Theorem 1). 
Let us say a few words about the relationships between the production and cost sharing games. Cost games, defined by assigning a cost to each coalition, are often considered as equivalent to those defined by a value function. Indeed it suffices to consider the opposite of the cost to obtain a game in characteristic form. However, here, the form of the constraints on each coalition also affects the game: in the production game the constraints write as $A x \leq b(S)$, and in a cost game as $A x \geq d(S)$, with $A$ a positive matrix in both cases. Thus transforming $c$ into $-v$ does not lead to a production game because the constraint remains $A x \geq d(S)$. As we will see, the complexity issues may substantially differ in the two cases.

\subsubsection{Partitioning games}

Partitioning Games extend the approach introduced by Shapley and Shubik [6] in their study of an assignment game (also called two-sided market). An assignment game describes a market, say a real estate market, in which there are two types of agents, prospective purchasers, the "buyers" and homeowners, the "sellers". Each buyer is interested in buying only one house. The $i$-th seller values his own house at $b_{i}$ dollars while the $j$-th buyer values the same house at $a_{i j}$ dollars. The important data are the "essential" coalitions, the pairs of buyer-seller, and the total value they derive by forming, here $v_{i, j}=a_{i j}-b_{i}$. The possible outcomes of the market specify which pairs of buyer-seller end up making a transaction and at what price. An outcome is in the core if no pair consisting of a buyer and a seller can make an arrangement that is more satisfactory to both than the given one. Shapley and Shubik [6] showed that the core is non-empty.

Partitioning Games extend the assignment game by considering a set of basic or 'effective' coalitions $\mathcal{E}$. The underlying idea is that the value to cooperation is derived only from these coalitions (as the pairs and the singletons in a two-sided market). ${ }^{2}$ Specifically, start with an initial assignment of values to the coalitions in $\mathcal{E}$ is given, denoted by $v_{j}$ for $S_{j}$. One derives a value to any coalition $S$, not necessarily in $\mathcal{E}$, by assuming it can split into a partition of several self-sufficient groups in $\mathcal{E}$ and achieve the sum of the values of the subsets in the partition. Thus, the value to $S$ is defined as the maximum value reached by its partitions into essential coalitions.

This construction is described as follows. Consider the incidence matrix $A$ with rows indexed by players and columns by elements in $\mathcal{E}$, where $A_{i, j}=1$ if $i$ belongs to $S_{j}$ and equal to 0 otherwise. A partition of $S$ into elements in $\mathcal{E}$ is described by a vector $x$ of 0,1 that assigns a value of 1 to each element in the partition: $\sum_{j} A_{i, j} x_{j}=1$ for each $i$ in $S$ and $\sum_{j} A_{i, j} x_{j}=0$ for each $i$ not in $S$. The first equations say that each individual is restricted to belong to exactly one coalition, and the second ones that no coalition contains an individual outside $S$. In matrix form these equations write $A x=\mathbb{1}_{S}$ in which $\mathbb{1}_{S}$ is the vector with components 1 on $S$ and 0 on $N-S$ (the indicator of $S$ ). This gives the value to $S$

$$
\begin{aligned}
& \pi_{I}(S)=\max \quad v^{t} x \\
& \text { s.t. } \quad A x=\mathbb{1}_{S} \quad x \geq 0, x \text { integers }
\end{aligned}
$$

\footnotetext{
${ }^{2}$ The approach has been followed by various authors Kaneko and Wooders [1], Boros, Gurvich and Vasin [3], Faigle and Kern [2], Le Breton, Owen, and Weber [8], and Demange [9] and [10].
} 
The subscript $I$ to $\pi$ denotes the integer requirement.

The core may be empty. For example take for $\mathcal{E}$ the whole set of coalitions and for $v$ the characteristic function of a super-additive game with an empty core. The value $\pi_{I}$ coincides with $v$ (because $v$ is super-additive), hence the core is empty. A main question is to find the sets $\mathcal{E}$ for which the core is always non-empty whatever the value $v$.

\subsection{Linear and Combinatorial Optimization Games}

In what follows, we combine the three types of inequalities constraints with the integer-valued requirement. We obtain the following versions of the games. The integer version represents "indivisibilities" in which combinations of activities are restricted to be integer valued. The matrix $A$ and the vectors have non-negative elements. ${ }^{3}$

In what follows $L P$ describes a standard linear program and $I L P$ the program obtained by requiring in addition the variable $x$ to be integer-valued, as described in full for the production game. The linear program $L P$ is often called the relaxation program of the corresponding program $I L P$.

Definition 1. Given $(v, A, b)$ the linear production $\nu_{L}$ game assigns to each $S, S \subseteq N$, the value equal to the value of the following linear program

$$
\begin{aligned}
& \nu_{L}(S)=L P(v, A, b, \leq ; S): \quad \max \quad v^{t} x \\
& \text { s.t. } A x \leq b(S) \quad x \geq 0
\end{aligned}
$$

and the integer production $\nu_{I}$ game is defined by assigning to each $S, S \subseteq N$, the value of the integer linear program where the components of $x$ are required to be integers

$$
\begin{aligned}
& \nu_{I}(S)=I L P(v, A, b, \leq ; S): \quad \max \quad v^{t} x \\
& \text { s.t. } A x \leq b(S) \quad x \geq 0, x \text { integers }
\end{aligned}
$$

Given $(v, A, b)$, the exact production game $\pi_{L}$ assigns to each $S, S \subseteq N$, the value

$$
\begin{aligned}
& \pi_{L}(S)=L P(v, A, b,=; S): \quad \max \quad v^{t} x \\
& \text { s.t. } A x=b(S) \quad x \geq 0
\end{aligned}
$$

and the integer exact production game $\pi_{I}$ assigns to $S$ the value $\operatorname{ILP}(v, A, b,=; S)$ of the program. Given $(c, A, d)$, the cost sharing game $\sigma_{L}$ assigns to each $S, S \subseteq N$, the cost defined by

$$
\begin{aligned}
& \sigma_{L}(S)=L P(c, A, d, \leq ; S): \quad \min \quad c^{t} x \\
& \text { s.t. } A x \geq d(S) \quad x \geq 0
\end{aligned}
$$

and the integer cost game $\sigma_{I}$ assigns to $S$ the value $I L P(c, A, d, \leq ; S)$.

\footnotetext{
${ }^{3}$ By convention, when a program is not feasible, the solution is defined to be $+\infty$ for minimization problems and $-\infty$ for maximization problems.
} 
Observe that for each $S \subseteq N$ we have $\nu_{L}(S) \geq \nu_{I}(S), \pi_{L}(S) \geq \pi_{I}(S)$, and $\sigma_{L}(S) \leq \sigma_{I}(S)$.

We now introduce our main concept.

\subsection{Universally Balanced Games}

The core of a game may be empty. A fundamental result is the Bondareva-Shapley theorem (Bondareva [11], Shapley [12]), which provides a characterization of the games with non-empty cores in terms of the balanced property. The game $\nu$ is balanced if for any set of non-negative real numbers $\gamma \in 2^{N} \rightarrow R_{+}$such that, for each $i \in N, \sum_{S: i \in S \subseteq N} \gamma(S)=1$, we have $\sum_{S \subseteq N} \gamma(S) v(S) \leq \nu(N)$. The core of $\nu$ is non-empty if and only if the game is balanced.

Universally balancedness bears on a class of games and asks that each game in the class is balanced, i.e., has a non-empty core. This concept has been used in various contexts, under various names, as will be illustrated in the examples of Section 4 . For a class defined by a set of parameters, as those just introduced, universally balancedness requires that each game is balanced independently of the numerical values taken by some or all of these parameters. To avoid repetition, let us give a generic definition.

Definition 2. A class of games parameterized by a set of parameters $T$ is said to be universally balanced (with respect to $T$ ) if for all values of $t$ in $T$, the core of the game is non-empty.

Our games are defined by multi-dimensional parameter and we may consider some components fixed. Consider the class of integer production games for example. A specific game in the whole class is defined by the numerical values taken by the parameters $(A, v, b)$. Anticipating, the core may be empty, that is, the whole class is not universally balanced. But we may keep $A$ fixed and allow $v$ and $b$ to vary. Thus, universally balancedness with respect to $(v, b)$ identifies the matrices $A$ that guarantee the core to be non-empty whatever the admissible values for $v$ and $b$.

\subsection{Computational Complexity}

We provide here a minimum necessary information for readers not familiar with the computational issues. It would be impossible to introduce computational complexity at this short treatment. We give a rather informal introduction to the most necessary ingredients for our discussion. Readers want to get more familiar with the topic should find a standard textbook, such as in Garey and Johnson [13].

The computational question is to design a step-by-step procedure to determine whether a certain property is satisfied or not, such as whether a given vector is in the core or not. A procedure that always produces a correct answer is called an algorithm.

Let $t(n)$ be a function of integer $n$. For an input of size $n$, if an algorithm produces the correct answer in no more than $t(n)$ operations, its complexity is said to be no more than time $t(n)$. Often $O(t(n))$ is used to represent the fact that the number of operations is always no more than $C * t(n)$ for some absolute constant $C>0$. A polynomial time function increases at a much slower pace than an exponential function. Taking into account this fact, algorithms that run in polynomial time are referred to as good algorithms by Edmonds [14]. Good algorithms with respect to succinctly represented games were proposed by Megiddo for finding nucleolus, another important solution concept in cooperative games [4]. The possibility of designing good algorithms has been proposed as a rationality consideration by Deng and Papadimitriou [15]. 
Unfortunately, it is not always possible to find a good algorithm. In evaluating the difficulty for computation of decision problems, the concept of Non-deterministic polynomial time (commonly referred to as NP) was introduced by Cook [16] to characterize a large class of computational problems. Informally, a problem is in NP if there is a polynomial time algorithm to VERIFY that the input to a decision problem (with YES-NO outcomes) belongs to the class of YES outcomes ${ }^{4}$. One may note that a good algorithm (polynomial time) for verification may not imply a polynomial time algorithm to find a solution. A problem is NP-hard if it can be used as a sub-procedure to find a solution for every problem in the class NP, in polynomial time and polynomial number of calls to the sub-procedure.

An NP-complete problem is one that is both in NP and NP-hard. Informally, it can be considered as the most difficult problem in the class of NP. Symmetrically, we can define co-NP-complete problems, which are decision problems for which its complement is NP-complete. Among the first proven NP-complete problems [16,17], the integer programming problem to find a solution to a set of linear inequalities with integrality constraints is a very useful benchmark NP-hard problem.

We consider games that have a succinct representation. It is well known that the linear programming problem with rational inputs and real variables is polynomial time computable. Thus there is a good algorithm which, given any subset $S$ of players, outputs the value $\nu_{L}(S)$ in polynomial time. On the other hand, some of our problems are integer constrained, and turn out to be complex from a computational point of view. ${ }^{5}$

\section{A General Result}

The first statement has been shown by Owen [5] for linear production games and trivially extends to other games. The second statement gives sufficient conditions under which the core of an integer game is non-empty.

\section{Theorem 1.}

(1) The class of linear production games is universally balanced: their cores are non-empty. The same property holds for exact production and cost sharing games.

(2) If the game value for $N$ in the integer production game, $\nu_{I}(N)$, is equal to the value $\nu_{L}(N)$, then the core of the integer production game is non-empty. The same property holds for exact production and cost sharing games: if $\pi_{I}(N)$ is equal to $\pi_{L}(N)$ (respectively $\sigma_{I}(N)$ is equal to $\sigma_{L}(N)$ ), then the core of the integer exact production (respectively integer cost sharing) game is non-empty.

(1) Owen showed that the balancedness condition holds for the production game. The same proof applies to exact production and cost sharing games.

(2) We give the proof for the production game. Other cases are similar. We know that the core for the production game $\nu_{L}$ is non-empty. Take an allocation in the core: $\sum_{i \in N} z_{i}=\nu_{L}(N)$ and $z(S) \geq \nu_{L}(S)$. Under the assumption $\nu_{L}(N)=\nu_{I}(N), z$ is feasible for the integer problem. Observe that we have $\nu_{L}(S) \leq \nu_{I}(S)$ for each $S \subseteq N$ : the allocation belongs to the core of $\nu_{I}$ as well.

The converse of part (2) of Theorem 1 is not true. Let us take the cost sharing game as an example. Assume that the condition stated in (2) holds, i.e., the solution to the integer linear program is also a

\footnotetext{
${ }^{4} \mathrm{~A}$ tricky part is that there are no time constraints to verify that the input belongs to the class of NO outcomes

${ }^{5}$ For an analysis of complexity issues in games without transferable utility, see Ballester [18].
} 
solution to its linear program relaxation. We show that a small perturbation of the demands does not modify the integer game, hence its core remains non-empty, but that it modifies the value of the relaxed version, making it different from the value of the integer version. Decrease the demands by a small constant $\epsilon>0: d_{k}^{i}$ is modified into $d_{k}^{i}=d_{k}^{i}-\epsilon$, and denote by $\sigma_{L}^{\prime}$ and $\sigma_{I}^{\prime}$ the new cost functions. Clearly, for any $S \subseteq N$, a feasible solution for $\operatorname{ILP}(c, A, d, \geq ; S)$ will still be feasible for $\operatorname{ILP}\left(c, A, d^{\prime}, \geq ; S\right)$ : $\sigma_{I}^{\prime}(S) \leq \sigma_{I}(S)$. Furthermore, for $\epsilon>0$ sufficiently small, there is no new integer feasible solution, so that $\sigma_{I}^{\prime}(S)$ is equal to $\sigma_{I}(S)$. Indeed, we may assume all numbers in $A, c$ and $d$ are integers (scaling them if necessary). Then making $\epsilon=0.5 /|N|$ ensures that the change in the cost for $S$ is bounded by a fractional number less than 1: no new integer feasible solution is introduced. Therefore, the game $\sigma_{I}^{\prime}$ coincides with $\sigma_{I}$ : its core is non empty.

Now, the relaxed linear program will in general have a reduced optimal value, in particular we have $\sigma_{L}^{\prime}(N)<\sigma_{L}(N)$. Therefore the value $\sigma_{I}^{\prime}(N)$ differs from $\sigma_{L}^{\prime}(N)$ and the core of the integer game is non-empty: the reverse claim of part (2) of Theorem 1 does not hold.

Special cases of the integer-constrained games for which the converse of part (2) of Theorem 1 holds have been considered. These games, which we call simple combinatorial optimization games, will be introduced and studied in more details in Section 4.

\section{Simple Combinatorial Optimization Games}

This section considers simple combinatorial optimization games (following the terminology of Bas van Velzen [19]). They are special cases of the integer-constrained productions/cost-sharing games. ${ }^{6}$

\subsection{Description of the Simple Games}

The coefficients in the matrix $A$ and the vectors $v, b, c$, and $d$ are restricted to be zero-one values. A further restriction, $m=n$, is often imposed as if each player controls exactly one type of (different) resources. Such a resource can be thought as himself as in the partitioning example of Section 2.1. We present this version here. Let us spell the interpretation of each game.

First consider a production game. A matrix $A$ made of 0 and 1 without null columns can be viewed as the incidence matrix of a collection $\mathcal{E}^{*}$ of subsets of $N$ defined as follows. Associate to each column the subset of $N: S_{j}=\left\{i, A_{i, j}=1\right\}$. The resource of coalition $S$ is $\mathbb{1}_{S}$. Observe that a nonnegative integer vector $x$ that satisfies the constraint is made of 0 or 1 . It can thus be interpreted as choosing columns, i.e., elements of $\mathcal{E}^{*}$. The constraint $A x \leq \mathbb{1}_{S}$ then reads as requiring that the coalition $S$ chooses disjoint elements in $\mathcal{E}^{*}$, each one included in $S$. This is referred to in the computational literature as a packing problem.

For the exact production game, $x$ represents a partition of $S$ into elements of $\mathcal{E}^{*}$ : the game is a partitioning game as defined in Section 2.1.

\footnotetext{
${ }^{6}$ The simple combinatorial optimization game has its origin in the matching game due to Shapley and Shubik [6]. Faigle and Kern extended it to partition games [2]. Deng, Ibaraki and Nagamochi studied packing and covering games, corresponding to simple production and cost-sharing games [20].
} 
For the cost sharing game, the constraints $A x \geq \mathbb{1}_{S}$ require that each player in $S$ belongs to at least one element of $\mathcal{E}^{*}$. The above inequality does not imply that the components of $x$ are 0 or 1 . However, the value of

$$
\begin{aligned}
& \min c^{t} x \\
& \text { s.t. } \quad A x \geq \mathbb{1}_{S}, x \geq 0, x \text { integers }
\end{aligned}
$$

is reached at a unit vector. To see this, note that any integral feasible $x$ can be replaced with a unit-integral solution without violating the constraints (8) by changing any integer value more than one by one, that is any $x_{i} \geq 1$ by $x_{i}=1$. Since this can only lower the cost, the program value coincides with that of

$$
\min _{\text {s.t. }} \quad A x \geq \mathbb{1}_{S}, x \in\{0,1\}^{p} c^{t} x
$$

Such a problem is referred to as a covering game.

For simple games the converse of part (2) of Theorem 1 is true. From [2,20], we have

Theorem 2. The core of a packing (respectively partitioning, respectively covering) game is non-empty if and only if the game value for $N$ of the integer linear program $\nu_{I}(N)$ (respectively $\pi_{I}(N)$, respectively $\sigma_{I}(N)$ ) is the same as the value $\nu_{L}(N)$ (respectively $\pi_{L}(N)$, respectively $\sigma_{L}(N)$ ) of the linear relaxation program.

We give here an intuitive proof based on the effective coalitions. In the packing problem consider the collection of subsets $\mathcal{E}^{*}$ associated with the incidence matrix $A$. Interpret the value $v_{j}$ as the value of the effective coalition $S_{j}$. The key point is that the blocking conditions on an allocation $z=\left(z_{i}\right)$ simplify into the requirement that $z \geq 0$ and each effective coalition $S_{j}$ receives at least $v_{j}: \sum_{i \in S_{j}} z_{i} \geq v_{j}$ each $j$. That these conditions are necessary in both games is clear since $S_{j}$ can obtain alone $v_{j}$ by choosing $x$ to be the unit vector $\mathbb{1}_{\{j\}}$ and the value to a singleton is non-negative. That they are sufficient is proved as follows. Let $z=\left(z_{i}\right)$ be a non-negative vector of $R^{N}$. Given $S$, for any $x \geq 0$ that satisfies $A x \leq \mathbb{1}_{S}$, we have the following inequalities: $\sum_{i \in S} z_{i} \geq \sum_{i \in S} z_{i}\left(\sum_{j, i \in S_{j}} x_{j}\right)=\sum_{j} x_{j}\left(\sum_{i, i \in S_{j}} z_{i}\right)$. Thus, for $z$ an allocation under which $S_{j}$ receives at least $v_{j}, \sum_{i \in S_{j}} z_{i} \geq v_{j}$ each $j$ implies $\sum_{i \in S} z_{i} \geq \sum_{j} x_{j} v_{j}$. Since $\nu_{L}(S)$ (respectively $\nu_{I}(S)$ ) is defined as the maximum of the right hand side over all $x \geq 0$ that satisfy $A x \leq \mathbb{1}_{S}$ (respectively that satisfies $A x \leq \mathbb{1}_{S}$ for $x \geq 0$ integers), we obtain $\sum_{i \in S} z_{i} \geq \nu(S)$ for $\nu$ given by $\nu_{L}$ or $\nu_{I}: S$ does not block in either game.

Thus, the no-blocking constraints are the same in the game with or without integer constraints. As for the game values for the whole set $N$, the value $\nu_{I}(N)$ is obtained by considering partitions of $N$ (super-additive cover) and the value $\nu_{L}(N)$ is obtained by considering balanced families of $N$. When the core of the integer production game is non-empty, we have $\nu_{L}(N) \leq \nu_{I}(N)$ by Shapley theorem [12], hence the two values coincide (since the reverse inequality $\nu_{L}(N) \geq \nu_{I}(N)$ always holds). Similar arguments hold for the partitioning and covering games.

The link between packing games and partitioning games is strong under some conditions on the collection $\mathcal{E}$ or equivalently on matrix $A$. Take a partitioning game defined by a collection of efficient subsets $\mathcal{E}$ that contains the singletons, and normalize the values of $v$ on singletons to 0 . Define $\mathcal{E}^{*}$ the 
collection without the singletons. Using the interpretation above on an incidence matrix yields that the partitioning game defined by $\mathcal{E}$ is equivalent to the packing game associated with the incidence matrix of $\mathcal{E}^{*}$. However, when $\mathcal{E}$ does not contain the singletons, a partitioning game is not a packing game.

\subsection{Universally Balancedness in Simple Games}

A simple game is characterized by the matrix $A$ and the vector defining the objective $v$ or $c$ (since the resource constraint is defined by the unit vectors). We consider here the universally balancedness property with respect to the objective, keeping $A$ fixed. To simplify the terminology, we simply say that $A$ is universally balanced for the packing game if the class of packing games defined by $A$ is universally balanced with respect to $v$, and similarly for partitioning and covering problems.

From the Theorems 1 and 2, the core of a packing game is non-empty if and only if its relaxation program has an integer optimal solution. Therefore, the packing game given $A$ is universally balanced if and only if the polyhedron $\left\{x \in R^{p}: A x \leq \mathbb{1}_{N}, x \geq 0\right\}$ is integral, i.e., all its extreme vertices have integer values. Similar results obtain for the partitioning games (Faigle and Kern [2], Kaneko and Wooders [1]) and the covering games. This is stated as follows.

Theorem 3. Let $A$ be a $(0,1) n \times p$ matrix, and $N=\{1, \ldots, n\}$.

$A$ is universally balanced for the packing game if and only if the polyhedron $\left\{x \in R^{p}\right.$ : Ax $\left.\leq \mathbb{1}_{N}, x \geq 0\right\}$ is integral.

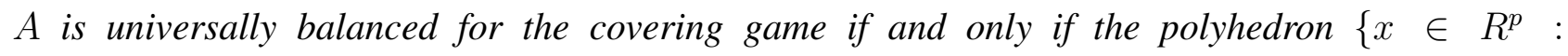
Ax $\left.\geq \mathbb{1}_{N}, x \geq 0\right\}$ is integral.

$A$ is universally balanced for the partition game if and only if the polyhedron $\left\{x \in R^{p}\right.$ : $\left.A x=\mathbb{1}_{N}, x \geq 0\right\}$ is integral.

Applying the work of Chudnovsky et al. [21] on perfect graphs and Chudnovsky et al. [22] for recognizing perfect graphs, we obtain the polynomial time solvability of determining whether the packing game is universally balanced.

Corollary 1. Determining whether A is universally balanced for the packing game can be done in polynomial time.

Contrary to the packing games, it is co-NP-complete to determine whether $A$ is universally balanced for the covering games.

Corollary 2. Determining whether $A$ is universally balanced for the covering game is co-NP-complete.

Recall that a problem is in co-NP if its complement problem is in NP. It is co-NP-hard if its complement is NP-hard.

For a partitioning game, it is important to distinguish whether singletons are effective. As we have seen, a partitioning game for which singletons are effective (i.e., matrix $A$ has a fully identity sub-matrix) can be written as a packing game. Then the problem can be decided in polynomial time. But, in general, the problem of determining whether $\left\{x: A x=\mathbb{1}_{N}, x \geq 0\right\}$ is integral is not known to have a polynomial time algorithm. 


\subsection{Simple Games on Graphs}

We specify even further the simple games by considering games derived from a graph. More precisely we consider for $A$ either the vertex-edge incidence matrix or its transpose the edge-vertex incidence matrix; the players will be either the vertices or the edges. The universally balanced properties for the associated combinatorial optimization games become particularly interesting especially for the complexity issues. Some applications are given in Section 4.4.

Consider a graph $G=(V, E)$ where $V$ denotes the set of vertices and $E$ the set of edges. There are $m$ vertices and $p$ edges.

The vertex-edge incidence matrix is the $m \times p$ matrix $A$ such that $A_{i, j}=1$ if $i \in V$ is an incident vertex of edge $j \in E$. That is, $j=(i, \ell)$ for some $\ell \in V$.

The edge-vertex incident matrix is the $p \times m$ matrix $B$ such that $B_{j, i}=1$ if $j \in E$ is an edge that contains vertex $i: B$ is the transpose of $A$.

A vertex-edge (respectively edge-vertex) incident matrix of a graph is characterized by the fact that each column (respectively row) has exactly two elements equal to 1 . Note that these matrices differ from the square incidence matrix indexed by the set of vertices that has a 1 if $i, j$ is an edge and a 0 otherwise.

Each matrix $A$ or $B$ define a different type of packing/covering/partition games. The set of players for $A$ is the set of vertices $V$, and for $B$ the set of edges $E$.

Packing Games Consider first the game with vertices as players and matrix $A$. Before describing the result, let us explain a little bit the game. We show that the problem faced by a coalition is to find a "maximum weighted matching". Hence we call such a game a maximum weighted matching game. The class is obtained by keeping the graph fixed and varying $v$.

Start with the whole set $V$. It must choose $x$ in $\{0,1\}^{E}$ that satisfies $A x \leq \mathbb{1}_{V}$. The vector $x$ can be interpreted as choosing a subset of edges $U$. The constraint $A x \leq \mathbb{1}_{V}$ reads that no vertex is incident to more than one edge. In other words no two edges in $U$ have a vertex in common: $U$ defines a matching (by matching the two vertices of each edge). Given weights $v$ on the edges, $V$ seeks for a maximum weighted matching, one for which the sum of the individual edges weights is maximum. Consider now a coalition $S$ of vertices. The constraint $A x \leq \mathbb{1}_{S}$ requires $U$ to be a matching within $S$.

By Theorems 2 and 3, the packing game defined by $A$ is universally balanced if and only if the values for the linear programs $L P(v, A, \leq)$ and $\operatorname{ILP}(v, A, \leq)$ coincide, which is equivalent to $\left\{x \in R^{E}\right.$ : $\left.A x \leq \mathbb{1}_{V}, x \geq 0\right\}$ is integral.

If there is an odd cycle, $\left(i_{1}, i_{2}, \cdots, i_{2 k+1}\right)$ for some $k \geq 1$, then setting $v_{j}=1$ for every edge $j$ of the cycle, and $v_{j}=0$ otherwise, will result in a fractional optimal value, $k+1 / 2$, for the linear program $L P(v, A, \leq)$. This value necessarily differs from the value of the integer program. Therefore, the core of the integer problem is empty for this particular weight function and universally balanced requires the graph to have no odd cycle, that is the graph must be bipartite. Conversely, if the graph is bipartite, there is always an integer optimal solution to the linear program by Konig's Theorem [23]. 
Theorem 4. The class of maximum weighted matching games defined on a graph is universally balanced if and only if the graph is bipartite.

Consider now the game with edges as players defined by the edge-vertex incident matrix. We show that the problems faced by coalitions are to find a maximum weighted independent vertex set. Hence we call such a game a maximum (weighted) independent vertex set game.

Similarly as above, $E$ chooses $y$ in $\{0,1\}^{V}$ that can be interpreted as choosing a set of vertices $U$. The constraint $B y \leq \mathbb{1}_{E}$ reads that no edge has its two vertices in $U$, or in other words no two vertices in $U$ are linked in $G$. The set $U$ is said to be an independent vertex set. Given weights $v$ on the vertices, one seeks for a maximum independent vertex set, one for which the sum of the individual vertex weights is maximum.

For a coalition of edges $S$, the constraint $B y \leq \mathbb{1}_{S}$ reads as no edge in $S$ has its two vertices in $U$ and an edge not in $S$ has no vertex in $U$. In other words, first delete all vertices that are incident to an edge not in $S$, and find an independent set in this reduced graph.

By Theorem 3, the game corresponding to $B$ is universally balanced if and only if $\left\{y \in R^{V}\right.$, $\left.B y \leq \mathbb{1}_{E}, y \geq 0\right\}$ is integral.

If there is an odd cycle, we choose one of the shortest in length, $\left(i_{1}, i_{2}, \cdots, i_{2 k+1}\right)$ for some $k \geq 1$. Let $v_{i}=1$ for every vertex of the cycle, and $v_{i}=0$ otherwise. One easily checks that the linear program $L P(v, B, \leq)$ has a fractional optimal value. Again, a necessary condition for the polyhedron to be integral is that there is no odd cycle on the graph, meaning that the graph must be bipartite. On the other hand, if the graph is bipartite, there is always an integer optimal solution to the linear program by Konig's Theorem [23].

Theorem 5. The class of maximum weighted independent vertex set games on a graph is universally balanced if and only if the graph is bipartite.

Covering Games The constraints for covering games are easier to understand than for packing games.

Start with the vertex-edge game. The constraints faced by a subset $S$ of $V$ ( $S$ possibly identical to $V$ ) are: $x$ in $\{0,1\}^{E}$ such that $A x \geq \mathbb{1}_{S}$. They require $S$ to choose a set of edges $U$ such each vertex in $S$ is incident to at least one edge in $U$. $U$ is called an edge covering set or edge cover. Given costs $c$ on the edges, one seeks for the minimum weighted edge cover, the one for which the sum of the cost of the edges is minimum. The terminology follows.

By Theorem 3, the covering game corresponding to $A$ is universally balanced if and only if $\left\{x \in R^{E}\right.$ : $\left.A x \geq \mathbb{1}_{V}, x \geq 0\right\}$ is integral. From the work of Ding, Feng and Zang [24] it follows that

Theorem 6. It is co-NP-complete to decide whether the class of minimum weighted edge cover games on a graph is universally balanced.

To decide whether the class is not universally balanced, one must find an input with answer NO, that is a non-integer vertex to the polytope, $\left\{x \in R^{E}: A x \geq \mathbb{1}_{V}, x \geq 0\right\}$ and a linear program with the vertex as the unique optimal solution, which proves that the problem is in NP. The co-NP-hardness proof is quite deep [24].

Consider now the edge-vertex game. The constraints faced by a subset $S$ of $E$ (possibly equal to $E$ ) are: $y$ in $\{0,1\}^{V}$ such that $B y \geq \mathbb{1}_{S}$. They require $S$ to choose a set of vertices $U$ such each edge in $S$ 
has at least one of its endpoints in $U . U$ is called a vertex cover (or vertex covering set), and given costs $c$ on the vertices, $S$ seeks for the minimum vertex cover.

The covering game corresponding to $B$ is universally balanced if and only if $\left\{y \in R^{V}, B y \geq \mathbb{1}_{E}\right.$, $y \geq 0\}$ is integral.

For any non-bipartite graph $G$, let $\left(i_{1}, i_{2}, \cdots, i_{2 k+1}\right)$ for some $k \geq 1$ be a minimum length odd cycle. Let $c_{i}=1$ for every vertex $i$ of the cycle, and $c_{i}=0$ for any other vertex $i$. Clearly, any integer solution requires at least $k+1$ vertices of the odd cycle which yields an optimal value at least equal to $k+1$. For the linear programming relaxation however, setting $y_{i}=1 / 2$ for the vertices on the cycle and $y_{i}=1$ for all other vertices yields a feasible solution with value $k+\frac{1}{2}$. Again, universally balanced requires the graph to be bipartite.

Conversely, for any bipartite graph, the minimum weighted vertex cover has the same value as its linear relaxation problem, by Konig's theorem [23]. Therefore, we have the following theorem:

Theorem 7. The class of minimum vertex cover games on a graph is universally balanced if and only if the graph is bipartite (and hence determinable in polynomial time). And when it is universally balanced, we can calculate the game value as well as finding an imputation in the core in polynomial time.

Partition Games Consider the partition game associated to the edge-vertex incident matrix $B$ of the graph. First of all, the polyhedron $\left\{y \in R^{V}: B y=\mathbb{1}_{E}, y \geq 0\right\}$ always has a feasible fractional solution: $y=\frac{1}{2} \mathbb{1}_{V}$. Therefore, for any $v$ the linear program relaxation, $\max \left\{v^{t} y, B y=\mathbb{1}_{E}, y \geq 0\right\}$, has a finite solution for any edge-vertex incident matrix $B$.

Now for a non-bipartite graph $G$, let $\left(i_{1}, i_{2}, \cdots, i_{2 k+1}\right)$ for some $k \geq 1$ be a minimum length odd cycle. Arguing as before let $v_{i}=1$ for every vertex of the cycle, and $v_{i}=0$ otherwise. One easily checks that the linear program $L P(v, B,=)$ has a fractional optimal value. It follows, by Theorem 2 , that a necessary condition for the partition game to be universally balanced is for the graph to be bipartite.

Now consider a bipartite graph. We show that the polyhedron $\left\{y \in R^{V}: B y=\mathbb{1}_{E}, y \geq 0\right\}$ is integral. It follows from Theorem 3 that $B$ is universally balanced for the partition game. Let $G=\left(V_{1}, V_{2} ; E\right)$ where $V_{1}$ and $V_{2}$ are independent, i.e., for any edge $(i, \ell) \in E$, we have $i \in V_{1}$ and $\ell \in V_{2}$. Without loss of generality, we may assume $G$ is a connected graph. For any solution $y$ that satisfies $B y=\mathbb{1}_{E}$, pick some vertex $i_{0} \in V_{1}$, and denote $y_{i_{0}}=y_{0}$. Then, the condition $\forall(i, \ell) \in E, y_{i}+y_{\ell}=1$ implies that $\forall i \in V_{1}, y_{i}=y_{0}$ and $\forall \ell \in V_{2}, y_{\ell}=1-y_{0}$. Therefore, $y$ is a combination of the two integral extreme points $\mathbb{1}_{V_{1}}$ and $\mathbb{1}_{V_{2}}$. We have thus proved

Theorem 8. The class of partition games defined by the edge-vertex incident matrix $B$ of a graph $G$ is universally balanced if and only if $G$ is bipartite.

Consider now the partition game with vertex-edge incident matrix $A$. A solution corresponds to a matching such that every vertex is matched, known as a perfect matching. In this case, the problem remains open. 


\subsection{Applications}

There are interesting applications involved with the game structure we discuss here.

First, there are standard applications of simple games in communication networks such as toll high ways, electronic cables, optical fibers. While nodes at both ends of a link derive some benefits for themselves, how to share the revenue can be modeled in a cooperative game framework as in [15]. Under this model, the value of a coalition is the sum of the edge values between all pairs of nodes in the networks. Similar interpretation can be given to cost games. Interesting research works have been developed along this direction.

Second, for the emerging social network systems over the Internet, simple game models also start to have potential real applications. Consider a graph representing friend relationships. Let an advertiser who wants to place advertisement on the social network so that each individual has an AD on his own web page or (not exclusive) on one of his friends' pages. The constraints can be written in terms of the incidence matrix of the graph (not the edge-vertex incidence matrix). The optimal solution to the covering problem will save advertiser's cost. Issues on how to share the revenue generated among the nodes is a very interesting problem.

Third, the maximum weighted matching game, on bipartite graphs, is a suitable model for the study of position markets (or matching markets) when buyers and positions are paired up [25-27]. The stability concept of the core and the universally balanced property explain to some extent the suitability of such market structures. There are also other potential applications in areas when resources are paired up to provide services.

Fourth, the minimum edge cover game can be a model for task assignment where each assignee is required to take up two tasks. The goal is to assign a minimum number of required persons watching over those tasks.

Finally, the maximum independent vertex set game can be used to model a survey game in a social network: individuals (which correspond to the nodes in the network) are paid to write a survey on a commercial products. To obtain a collection of independent opinions, we may want to collect opinions of people who are not related to each other. The weight represents an a priori evaluation of how informative a node will be.

Within the cooperative game framework over the Internet, more potential applications of our models of simple games are out there. A summary of what have been achieved in the past would be a benchmark we could build the solid theoretical foundation for the future.

\section{Universally Balanced Property with respect to Resources Constraints}

Here the matrix $A$ and the value $v$ are fixed, and $b$ is allowed to vary. Note that the property of universally balanced with respect to $b$ implies that the core for every subgame of the problem is non-empty, a property called totally balanced. 
Theorem 9. Linear production games $(v, A)$, cost sharing games $(c, A)$, partitioning games are universally balanced (hence totally balanced).

If the linear program has an optimal integer solution for all integer $b$, the problem always has a non-empty core (so do all its subgames). Such situations are related closely to the concept of total dual integral of Edmonds and Giles [28].

Definition 3. The linear system $\{y: y A \geq v, y \geq 0\}$ is total dual integral (TDI) if $\max \{v x$ : $A x \leq b, x \geq 0\}$ has an integer optimal solution for all integer vector $b$ whenever there is $a$ finite solution.

Theorem 10. If $\{y: y A \geq v, y \geq 0\}$ is a TDI system, then $(v, A)$ is universally balanced.

The same result can be derived for the linear cost sharing games as well as linear partition games.

It is not known whether the converse of Theorem 10 is true. It is an interesting problem to fully characterize the relationship between them.

\section{Concluding Remarks}

The universally balanced property identifies structural properties in cooperative games that guarantee the non-emptiness of the core, therefore, allows for stable or "group-rational" solutions in distributing social benefit to all the group members. The implementation issue of finding such solutions in presence of a large number of participants, measured in terms of computational complexity, is also a very actively studied area. Our discussion reviews some of the essential elements along this direction. Several open problems are pointed out.

The field has witnessed a strong interplay of game theory, combinatorial optimization as well as computational complexity in deriving understandings of the underlying principles. In the age of the Internet, and its applications such as social networks, cooperative games and structural results presented here will have a much more important role to play. We have introduced several interesting examples of such nature. We believe in times to come, the field will exhibit more influence in theory building for the emerging application areas. In presenting such a limited survey, we hope to bring together the theoretical methodologies and results that have been developed in different fields and groups, to provide a reference point for further research work to have a complete view of the foundations that are already laid down over the years.

\section{References and Notes}

1. Kaneko, M.; Wooders, M. Cores of partitioning games. Math. Soc. Sci. 1982, 3, 313-327.

2. Faigle, U.; Kern, W. Partition games and the core of hierarchically convex cost games. Universiteit Twente, Faculteit der Toegepaste Wiskunde, Memorandum. 1995, 1269.

3. Boros E.; Gurvich, V.; Vasin, A. Stable families of coalitions and normal hypergraphs. Math. Soc. Sci. 1997, 34, 107-123.

4. Megiddo, N. Computational complexity and the game theory approach to cost allocation for a tree. Math. Oper. Res. 1978, 3, 189-196. 
5. Owen, G. On the core of linear production games. Math. Program. 1975, 9, 359-370.

6. Shapley, L. S.; Shubik, M. The assignment game I: The core. Int. J. Game Theor. 1971, 1, 111-130.

7. Demange, G.; Wooders, M. Group Formation in Economics: Networks, Clubs and Coalitions; Cambridge University Press: Cambridge, UK, 2005.

8. Le Breton, M.; Owen, G.; Weber, S. Strongly balanced cooperative games. Int. J. Game Theor. 1992, 20, 419-427.

9. Demange, G. Intermediate preferences and stable coalition structures. J. Math. Econ. 1994, 23, 45-58.

10. Demange, G. On group stability in hierarchies and networks. J. Polit. Econ. 2004, 112, 754-778.

11. Bondareva, O. Some applications of linear programming methods to the theory of cooperative games. Problemy Kybernetiki 1963, 10, 119-139; In Russian.

12. Shapley, L.S. On balanced sets and cores. Nav. Res. Logist. Quart. 1967, 14, 453-460.

13. Garey, M.R.; Johnson, D.S. Computers and Intractability: A Guide to the Theory of NP-Completeness; W. H. Freeman and Company: San Francisco, CA, USA, 1979.

14. Edmonds, J. Paths, trees, and flowers. Can. J. Math. 1965, 17, 449-467.

15. Deng, X.; Papadimitriou, C. On the complexity of cooperative game solution concepts. Math. Oper. Res. 1994, 19, 257-266.

16. Cook, S.A. The Complexity of Theorem-Proving Procedures. In Proceedings of the Third Annual ACM Symposium on Theory of Computing, Shaker Heights, OH, USA, 1971; pp. 151-158.

17. Karp, R.M. Reducibility among combinatorial problems. In Complexity of Computer Computations; Miller, R.E., Thatcher, J.W., Eds.; Plenum: New York, NY, USA, 1972; pp. 85-103.

18. Ballester, C. NP-completeness in Hedonic Games. Games Econ. Behav. 2004, 49, 1-30.

19. Van Velzen, S. Simple combinatorial optimisation cost games. In CentER Discussion Paper; Center for Economic Research, Tilburg University: Tilburg, The Netherlands, 2005; RePEc:dgr:kubcen:2005118.

20. Deng, X.; Ibaraki, T.; Nagamochi, H. Algorithmic aspects of the core of combinatorial optimization games. Mathe. Oper. Res. 1999, 24, 751-766.

21. Chudnovsky, M.; Robertson, N.; Seymour, P.D.; Thomas, R. The strong perfect graph theorem. Ann. Math. 2006, 164, 51-129.

22. Chudnovsky, M.; Cornujols, G.; Liu, X.; Seymour, P.; Vuskovic, K. Recognizing Berge graphs. Combinatorica 2005, 25, 143-186.

23. König, D. Gráfok és alkalmazásuk a determinánsok és a halmazok elméletére. Matematikai és Természettudományi Értesitö 1916, 34, 104-119.

24. Ding, G.; Feng, L.; Zang, W. The complexity of recognizing linear systems with certain integrality properties. Math. Program. 2008, 114, 321-334.

25. Demange, G.; Gale, D.; Sotomayor, M. Multi-item auctions. J. Polit. Econ. 1986, 94, 863-872.

26. Edelman, B.; Ostrovsky, M.; Schwarz, M. Internet advertising and the generalized second price auction: Selling billions of dollars worth of keywords. Amer. Econ. Rev. 2007, 97, 242-259.

27. Varian, H. Position auction. Int. J. Ind. Organ. 2007, 25, 1163-1178.

28. Edmonds, J.; Giles, R. Total dual integrality and integer polyhedra. In Progress in Combinatorial Optimization; Pulleyblank, W., Ed.; Academic Press: London, UK, 1994; pp. 117-131. 
29. Scarf, H.E. The core of an n-person game. Econometrica 1967, 35, 50-69.

(c) 2010 by the authors; licensee MDPI, Basel, Switzerland. This article is an open access article distributed under the terms and conditions of the Creative Commons Attribution license http://creativecommons.org/licenses/by/3.0/. 